\title{
An Autonomous Surveillance Vehicle for People Tracking
}

\author{
C. Piciarelli, C. Micheloni, and G.L. Foresti \\ Department of Computer Science, University of Udine, \\ Via delle Scienze 206, 33100 Udine, Italy
}

\begin{abstract}
In this paper, the problem of the surveillance and the security of indoor environments is addressed through the development of an autonomous surveillance vehicle (ASV). The ASV has been designed to perform the object detection by adopting an image alignment method followed by a change detection operation. Hence, in addition to the classical robotic tasks (e.g., navigation and obstacle avoiding), the tracking of objects (e.g., persons) moving in an indoor environment is considered. The tracking procedure allows the ASV to maintain the interesting objects in the centre of the image, and in specific cases to focus the image acquisition on particular parts of the object (e.g., face of a person, etc.) for recognition purposes. Experimental results have been performed on different real scenarios where no objects moves inside the monitored scene and where at least one moving object is into the scene.
\end{abstract}

\section{Introduction}

The research work done in the last years on the field of mobile vision focused on many fields: aircrafts [2], autonomous underwater vehicles (AUV) [11] and autonomous guidance vehicles (AGV) moving on the ground [8, 13]. Certainly the work made in guidance of ground vehicle represents the great part of the research in context of mobile navigation where great interest has been pointed on indoor environments. From the first systems like those proposed by Giralt et al. in [6] and by Moravec in [10] many systems have been developed. For greater details on indoor navigation systems the reader is referred to [4]. Recently, some researchers have analyzed the problem of employing AGVs for surveillance purposes in both outdoor and indoor environments. In [3], Lipton et al. employed an airborne platform and ground vehicles to develop a multicamera system to monitor activities in cluttered outdoor environments using a distributed network of fixed and mobile sensors. In this context, the employment of mobile robots equipped with specific visual sensors for surveillance purposes can become an important tool.

In this paper, we have focused our attention to the problem of the surveillance and the security of indoor environments and, to address this objective, an autonomous surveillance vehicle (ASV) has been designed and developed. The ASV is able to perform, in addition to the classical robotic tasks (e.g., navigation in an indoor environment by avoiding obstacles), the tracking of mobile objects (e.g., persons). The selection of the target object to be tracked can be decided by a remote operator or autonomously by the system itself in the case of the presence of an alarm. The tracking procedure allows the system to maintain the objects of interest in the centre of the image, and in specific 
cases to localise particular parts of the object (e.g., face of a person) for recognition purposes.

The proposed ASV is able to detect moving objects by means of a direct method [14] and by computing the affine transform for the alignment of the two consecutive frames. An iterative and a multiresolution alignment techniques have been interleaved to increase the reliability of this task. Moreover, to overcome the problem of parallax an active zoom based technique has been introduced to increase the alignment performance in hallways context. Once the objects have been detected, a tracking module [5], based on a Kalman Filter, is able to estimate the position of the objects at the next time instant. Such estimations are used by the control module to autonomously change the position of the ASV in order to maintain the objects of interest as close as possible to the centre of the image.

\section{Motion Detection}

The principal activity of this module is the detection of mobile objects inside the scene. The problem consists in the identification of the motion due to the camera actions and of the real motion of the objects. The proposed solution is given by applying a direct method [14] to compute the affine transform for the alignment of two consecutive frames.

From the equation of the optical flow [9] and by considering the equation of the affine displacements, the following equation of the affine flow holds:

$$
I_{x}\left(a_{11} x+a_{12} y+a_{13}\right)+I_{y}\left(a_{21} x+a_{22} y+a_{23}\right)+I_{t}
$$

where $I_{x}, I_{y}$ and $I_{t}$ are the spatial and temporal derivatives of the brightness intensity.

From (1), it is possible to define a linear system $\mathbf{A x}=\mathbf{b}$ by considering each pixel of the image. Therefore, in order to reduce its computational complexity, we have adopted the form $\mathbf{A}^{T} \mathbf{A x}=\mathbf{A}^{T} \mathbf{b}$ which yields to (2) where only the upper section of the symmetric matrix $\mathbf{A}^{T} \mathbf{A}$ has been shown.

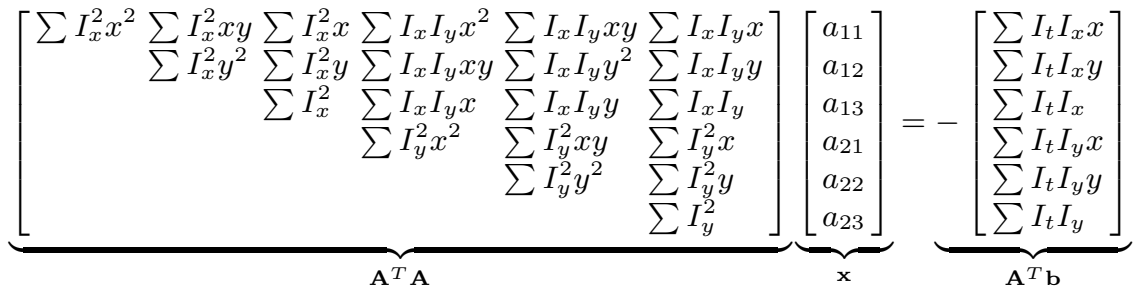

Adopting such equation for the affine transform computation, requires to solve the problems involved by the optical flow. First, when both spatial derivatives are equal to zero an adaptive thresholding technique [12] allows to discharge the $80 \%$ of the pixels by considering only those whose gradient is greater than a threshold $T_{p g}\left(\nabla I(x, y) \geq T_{p g}\right)$. Instead, to sidestep the inability of the optical flow method to work on large displacements, we have adopted two techniques: a) Iterative alignment and b) Multiresolution alignment. Two heuristics have been studied to speed up the iterative alignment process compared to the Newton-Raphson scheme. The first consists in the factorization of the 
equation (2) by adopting the method proposed by Cholesky. This resulted in a reduction of the complexity of a factor 2 . The second is given by introducing a time correlation of the alignment between two consecutive frames. Precisely, the iterative process is initialised with the parameters of the affine transform computed for the previous frame. The proposed solution with the adopted heuristics is presented in Algorithm 1

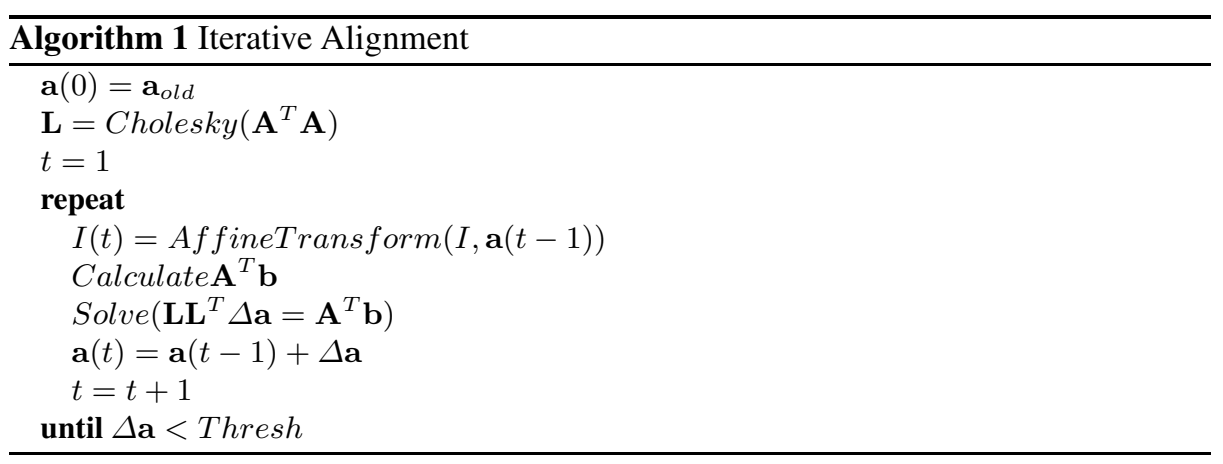

Since the iterative method, adopted in context of wide displacement between consecutive frames, requires an high number of iterations, we propose an alignment method based on a multiresolution approach. Each frame at different resolutions, determined by using a logarithmic scaling factor,is considered. The parameters of the affine transform are computed first at the highest level (i.e. the level corresponding to the image with lower resolution) then on the lowers. At each level a new iterative process for the alignment computation is started by using the partial solution reached at the previous level.

Besides this, optical flow implies to overcome a further problem given by the computation of the affine flow on pixel belonging to moving objects. Precisely, exploiting such pixels in the computation of the affine transform would result in a wrong alignment. Hence, in order to reduce their affect on the result or to give them a minor weight into the equation (2), we have adopted a robust estimator. The choice of the iteratively reweighed least square (IRLS) [1] as robust estimator, allows to maintain the structure of the described method. With this estimator the linear system in equation (2) becomes:

$$
w \mathbf{A}^{T} \mathbf{A} \mathbf{x}=w \mathbf{A}^{T} \mathbf{b}
$$

where the weights $w_{j}$ are computed by the estimator as follows:

$$
w_{i}=w\left(x_{i}\right)=\frac{\rho^{\prime}(x)}{2 x_{i}}
$$

where $\rho$ is the function of the estimator and $x_{i}$ is the residual of the pixel $i$. The function selected as estimator is the

$$
\rho(x, \sigma)=\log \left(1+\frac{1}{2}\left(\frac{x}{\sigma}\right)^{2}\right)
$$

with $\sigma$ equal to the scaling factor. 

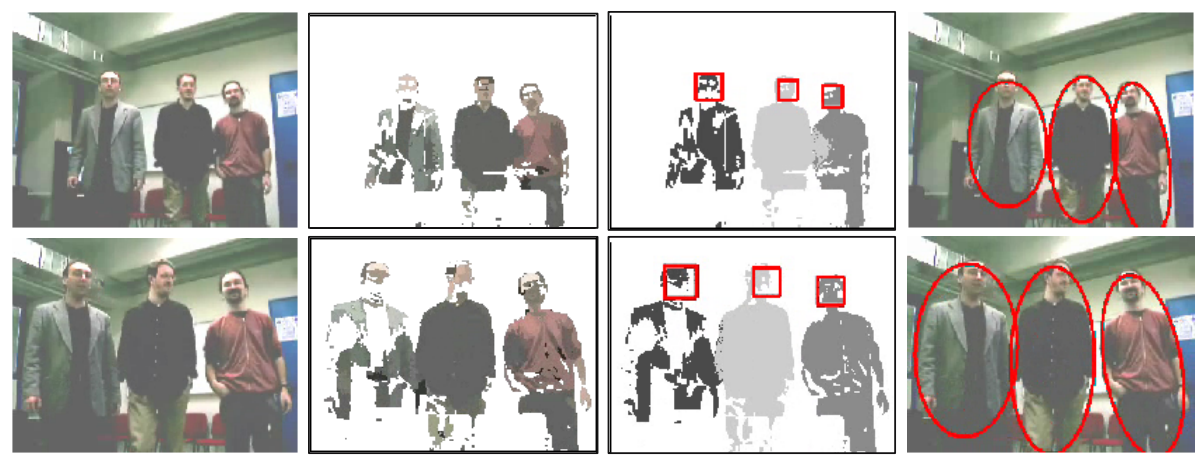

Fig. 1. People detection inside a group. First column: original sequence, second column: the result of the region identification algorithm, third column: face and body detection, fourth column: bounding ellipses for each person composing the group.

Once the alignment phase has been successfully executed, a change detection operation is applied on the two considered frames in order to detect the moving pixels. The resulting binary image is processed to compute the bounding ellipses of all moving blobs.

If the detected blob is composed by a group of people, in order to segment different persons a separation algorithm was developed. The proposed method searches the faces inside the blobe using a face detector based on a cascade of boosted classifiers working with Haar-like features, as described in [16]. The moving blob is then segmented in different regions of similar colours, and finally the regions are associated to the nearest detected face in order to identify the body of each person. The whole process is shown in figure 1

\section{Active Zoom}

In this section it will be discussed how the zoom control affects the proposed ASV system. Before studying how the ASV performances can be improved by the active control of camera zoom, it must be shown that the proposed system can control it. Actually many tracking systems cannot use the zoom because of the algorithms used for image alignment (e.g., it is the case of correlation-based systems) since correlation is not scale independent.

In the simple pinhole camera model, zoom is modelled as the change of the distance of the image plane from the pinhole. The zoom thus moves each pixel at coordinates $(x, y)$ in the new position $\left(x^{\prime}, y^{\prime}\right)$, where

$$
(x, y)=\left(f \frac{X}{Z}, f \frac{Y}{Z}\right) \quad\left(x^{\prime}, y^{\prime}\right)=\left(f^{\prime} \frac{X}{Z}, f^{\prime} \frac{Y}{Z}\right)
$$

where $(X, Y, Z)$ are the coordinates of the point in the world projected in the considered pixel and $f, f^{\prime}$ are the two focal lengths before and after the zoom. This means that $x^{\prime}=x f^{\prime} / f$ and $y^{\prime}=y f^{\prime} / f$, or, written in homogeneous notation 


$$
\left[\begin{array}{ccc}
f^{\prime} / f & 0 & 0 \\
0 & f^{\prime} / f & 0 \\
0 & 0 & 1
\end{array}\right]\left[\begin{array}{l}
x \\
y \\
1
\end{array}\right]=\left[\begin{array}{c}
x^{\prime} \\
y^{\prime} \\
1
\end{array}\right]
$$

which is an affine transform with parameters $a_{11}=a_{22}=f^{\prime} / f$. Since the proposed system, as shown in section 2, is based on an affine motion model, it can successfully handle the image warping introduced by zoom. Actually more complex zoom models exist, but they show that the error introduced with the pinhole approximation is small and non noticeable if the distance of the object from the optics is much bigger than the focal length [15]. The zoom can also be actively used to improve the quality of tracking and to allow the use of high-level surveillance tasks. Murray [7] distinguishes between two purpose-driven zoom and reactive zoom and claims that the zoom can improve the performance of the system.

In our solution, the forward translation of the camera introduces a parallax movement that cannot be modelled as an affine transformation. If the camera is translated by a distance $c$, the point $(x, y)=(f X / Z, f Y / Z)$ moves to

$$
\left(x^{\prime}, y^{\prime}\right)=\left(f \frac{X}{Z-c}, f \frac{Y}{Z-c}\right)=\left(x \frac{Z}{Z-c}, y \frac{Z}{Z-c}\right)
$$

The scaling factor $Z /(Z-c)$ is potentially different for every image point, since it depends from the distance $Z$ of the point from the camera, but it can be approximated as constant (and consequently affine) when $Z>>c$. Since $c$ is the translation of the camera between the acquisition of two consecutive frames, this impose an upper limit to the camera speed. If the pursued object is moving faster than the camera, then the active zoom can be used to keep the object's area constant, thus allowing a better object detection. The use of zoom also improves the alignment quality when the camera rotates in pan or tilt direction. In this case, the image transformation is not affine (it is actually projective), but can safely be approximated as an affine translation when the focal length, and consequently the zoom level, is big enough.

\section{Experimental Results}

The proposed method has been tested on sequences acquired in the hallways of the University building. Several experiments have been executed following a strategy that involves an incremental complexity for the tests. The sequences used for the tests have been acquired by a Cohu 3812 CCD camera mounted on a Robosoft Pioneer 2-DXe mobile indoor platform and are characterised by images of $320 \mathrm{X} 240$ pixels. The tests have been performed on a laptop equipped with an Athlon $2 \mathrm{GHz}$ processor and 256MByte of RAM. To evaluate the motion detection module a first test has been executed on sequences with no moving objects. In order to evaluate the precision of the alignment the following measure has been adopted:

$$
E=\frac{\sum_{i} g_{i} \log p_{i}}{\sum_{i} \log p_{i}}
$$

where $g_{i}$ are the grey values and $p_{i}$ the number of pixels with intensity value $g_{i}$. The value $E$ has been computed on sequences characterised by a simple rotational movement, translational movement, longitudinal motion and by a combination of all these 


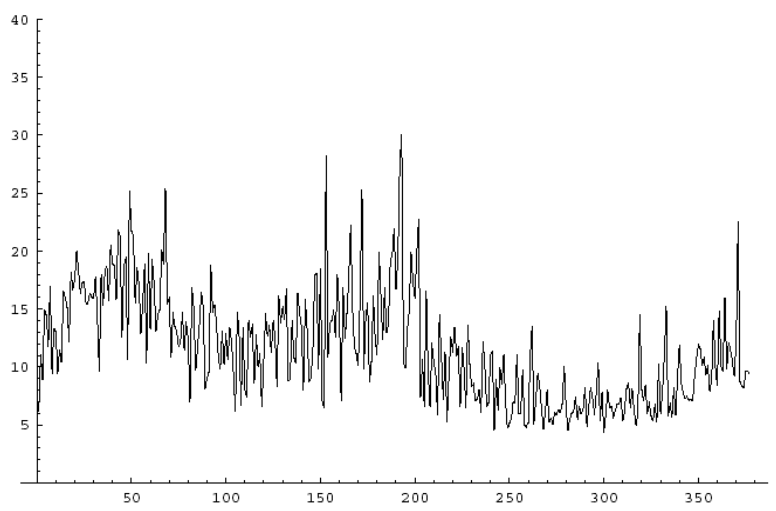

Fig. 2. Motion detection on sequences without moving objects in the scene. On the left some frames of a test sequence are shown, on the right the values of the error for the represented sequence are plotted. The mean value of the error $\mathrm{E}$ is 11.60.

types. The experiments significance has been increased by introducing different velocities on the mobile platform. The obtained results have pointed out a mean value for the error $E$ equal to 12.18 . Moreover, we have computed the number of iterations requested by our algorithm to converge and the average value, over all the test sequences, is equal to 1.56. Both values represent a good result by showing how the developed system performs a good alignment in few iterations. In Figure 2, the chart of the computed error for a test sequence is shown. To test the motion detection module, a second phased based on sequences presenting at least a moving object has been executed. It has been shown a good behaviour of the system as the object detection has been successful in the $97.4 \%$ of the frames. It is worth noting that the $2.6 \%$ of the cases in which the detection failed are related to spots and not to continuous frames. For these tests the average value of the error $E$ is equal to 12.58 that is really close to the performances obtained in context of no moving objects. Regarding the number of iteration needed to converge the value 1.92 is once again very close to the previous tests value. To test the performance of the object tracking module, ten people have been asked to process the test sequences in order to define the ground truth position of the moving person barycentre. Then, the ground truth position for each frame has been computed as mean of the position marked by each person. The results obtained in context of tangent motion (objects moving orthogonally to the optical axis) showed that our method reached a good performance level. For these kinds of sequences, the mean error in the estimation of the barycentre is equal to 20.85 pixels. This error is principally due to the vertical component while if we consider only the estimation error performed on the horizontal axis the performance increases to 12.18 pixels. We can consider this a good behaviour if we analyse the motion involved in this type of sequences. Regarding the radial motion (moving object going toward to or faraway from the camera) the global error has been equal to 16.43 pixels that is lower than the error performed on the previous sequences. Also in this context, by considering only the horizontal component, the error decrease to only 9.66 pixels. Globally the module of object tracking has supplied good results allowing to maintain the object at the centre of the image, or in its closer region, for 


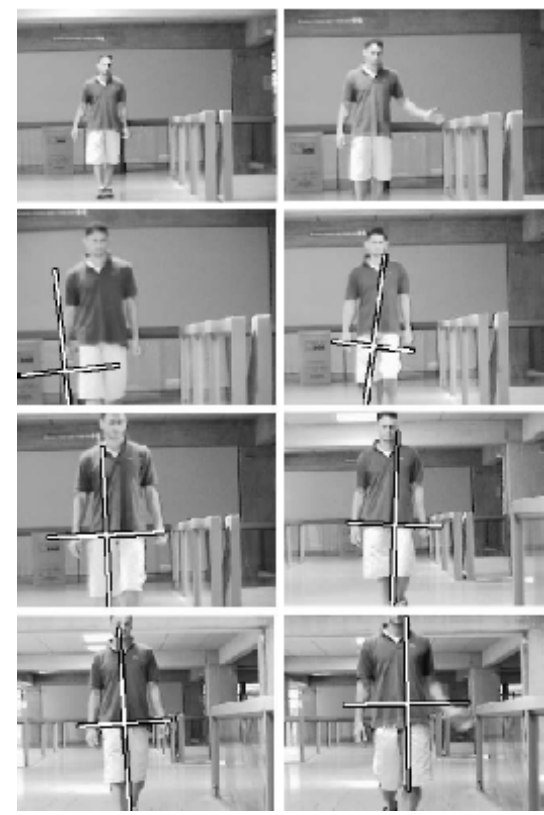

(a)

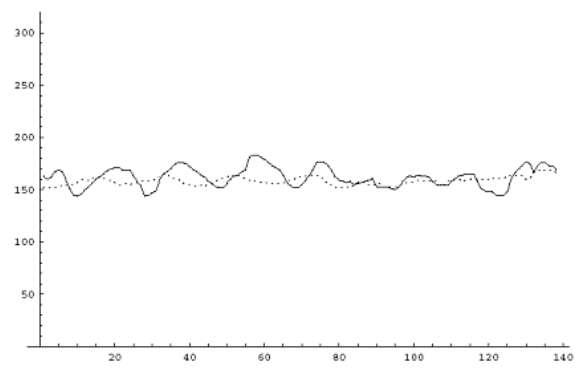

(b)

Fig. 3. Example of object tracking. On the left some frames of the sequences are shown, while in the right side the chart of the barycentre position is shown. The chart plot the horizontal position of the barycentre as marked by the testers (dotted line) and the estimated value computed by the tracking module (continuous line).

the majority of the frames belonging to the test sequences. An example of the results obtained by the proposed system can be seen in Figure 3

\section{Conclusions}

In this paper, the use of an autonomous vehicle, called autonomous surveillance vehicle (ASV), for surveillance and monitoring of indoor environments has been presented. The ASV has been specifically designed for help a remote operator in the work of monitoring wide indoor areas. As it has been shown, it is able to move around a specific indoor environment (e.g., a building) and to track mobile persons. To achieve such results the system is composed by two main modules:a) object detection and b)object tracking. The former allow to detect moving object, by first aligning two consecutive frame by interleaving multiresolution and iterative methods and therefore applying a change detection technique to highlight pixels belonging to mobile objects. The latter, is based on a Kalman Filter in which the prediction phase has been modified to take into account the previous results in the detection. Several experiments on indoor sequences demonstrate that the proposed ASV is able to detect the motion inside the monitored scene, then to perform a good identification of the mobile objects. Finally, such objects can be tracked with an accuracy that allows to maintain the objects inside the field of view. 


\section{References}

1. M. Black and A. Rangarajan. On the unification of line processes, outlier rejection, and robust statistics with applications to early vision. International Journal of Computer Vision, 19(1):57-92, 1996.

2. I. Cohen and G. Medioni. Detecting and tracking moving objects in video from and airborne observer. In Procceddings of the IEEE Image Understanding Workshop, pages 217-222, Manterey,CA, November 1998.

3. R.T. Collins, A.J. Lipton, H. Fujiyoshi, and T. Kanade. Algorithms for cooperative multisensor surveillance. In Proceedings of the IEEE, volume 89, pages 1456-1477, October 2001.

4. G.N. DeSouza and A.C. Kak. Vision for mobile robot navigation: A survey. IEEE Trans. on Pattern Analysis Machine Intelligence, 24(2):237-267, 2002.

5. G.L. Foresti and C. Micheloni. A robust feature tracker for active surveillance of outdoor scenes. Electronic Letters on Computer Vision and Image Analysis, 1(1):21-36, 2003.

6. G. Giralt, R. Sobek, and R. Chatila. A multi-level planning and navigation system for a mobile robot; a first approach to hilare. In Proceedings of International Joint Conference of Artificial Intelligence, volume 1, pages 335-337, 1979.

7. E. Hayman, T. Thorhallsson, and D. Murray. Zoom-invariant tracking using points and lines in affine views: an application of the affine multifocal tensors. In International Conference on Computer Vision, pages 269-277, 1999.

8. M. Herbert, C. Thorpe, and S. Stenz. Intelligent Unmanned Ground Vehicles: Autonomous Navigation Research at Carnegie Mellon. Kluwer Academic, 1997.

9. B. K. P. Horn and B. G. Schunk. Determining optical flow. Artificial Intelligence, 17:185203, 1981.

10. H.P. Moravec. The standford cart and the cmu rover. Proc. IEEE, 71(7):872-874, 1983.

11. J. Rosenblatt, S. Williams, and H.Durrant-Whyte. Behavior-based control for autonomous underwater exploration. In Proceedings of the IEEE International Conference on Robotics and Automation, San Francisco,CA, April 2000.

12. P. Rosin. Thresholding for change detection. In Proceedings of IEEE International Conference on Computer Vision, pages 274-279, Bombay India, 1998.

13. B. Southall, T. Hague, J.A. Marchant, and B.F. Buxton. Vision-aided outdoor navigation of an autonomous horticultural vehicle. In Proceeding of the first International Conference on Vision Systems, 1999.

14. G. P. Stein and A. Shashua. Model-based brightness constraints: on direct estimation of structure and motion. IEEE Trans. on Pattern Analysis Machine Intelligence, 22(9):992$1015,2000$.

15. B.J. Tordoff and D.W. Murray. Reactive control of zoom while tracking using perspective and affine cameras. IEEE Trans. on Pattern Analysis Machine Intelligence, 26(1):98-112, 2004.

16. P. Viola and M. J. Jones. Rapid object detection using a boosted cascade of simple features. In IEEE CVPR, 2001. 